\title{
Low emittance design of the electron gun and the focusing channel of the Compact Linear Collider drive beam
}

\author{
M. Dayyani Kelisani, ${ }^{1,2}$ S. Doebert, ${ }^{1}$ and M. Aslaninejad ${ }^{2}$ \\ ${ }^{1}$ European Organization for Nuclear Research (CERN), BE Department, \\ CH-1211 Geneva 23, Switzerland \\ ${ }^{2}$ Institute for Research in Fundamental Sciences (IPM), School of Particles and Accelerators, \\ P.O. Box 19395-5531 Tehran, Iran
}

(Received 14 November 2016; published 27 April 2017)

\begin{abstract}
For the Compact Linear Collider project at CERN, the power for the main linacs is extracted from a drive beam generated from a high current electron source. The design of the electron source and its subsequent focusing channel has a great impact on the beam dynamic considerations of the drive beam. We report the design of a thermionic electron source and the subsequent focusing channels with the goal of production of a high quality beam with a very small emittance.
\end{abstract}

DOI: 10.1103/PhysRevAccelBeams.20.043403

\section{INTRODUCTION}

The compact Linear Collider (CLIC) project at CERN aims at building an electron-positron linear collider with a center of mass energy of $3 \mathrm{TeV}$ [1]. Figure 1 shows the general layout of the CLIC with a total length of $48 \mathrm{~km} \mathrm{[2].}$

For CLIC a two beam scheme has been developed in which a high current drive beam is used to efficiently power the main beam at the very high accelerating frequency of $12 \mathrm{GHz}$. The drive beam is generated in the drive beam injector with 4.2 A current, $140 \mu$ s total pulse duration, $50 \mathrm{~Hz}$ repetition rate and $0.5 \mathrm{GHz}$ bunch repetition frequency. A fully loaded linac operating at $1 \mathrm{GHz}$ is used to accelerate the drive beam to $2.4 \mathrm{GeV}$ followed by a series of delay lines and combiner rings allowing to increase the bunch repetition frequency to $12 \mathrm{GHz}$ and the beam current to $101 \mathrm{~A}$. There are two options for the drive beam injector: a high current thermionic gun with a sophisticated bunching system [3] or a high current radio frequency gun [4-5]. In the present work we will, however, focus only on the first option which is the CLIC baseline. Figure 2 shows the layout of the CLIC drive beam injector which starts from a high current thermionic gun. After the beam is generated in the electron source, it would be bunched and accelerated using a bunching system and an accelerating structure. The bunching system starts with three subharmonic bunchers (SHBs) at a frequency of $0.5 \mathrm{GHz}[6]$. The subharmonic bunchers are responsible for the initial bunching. They also provide the phase coding required later on for the bunch multiplication scheme. Downstream from the SHBs, a

Published by the American Physical Society under the terms of the Creative Commons Attribution 4.0 International license. Further distribution of this work must maintain attribution to the author(s) and the published article's title, journal citation, and DOI. prebuncher (PB) [7] and a traveling wave buncher (TWB) [8] both operating at $1 \mathrm{GHz}$ are situated.

At the end of the bunching system the average energy of the electrons reaches up to $2.38 \mathrm{MeV}$. Further acceleration up to $11 \mathrm{MeV}$ is provided through two identical fully loaded accelerating structures ACC-1 and ACC-2 (see Fig. 2), operating at $1 \mathrm{GHz}$ frequency [9]. The whole injector is located in a long solenoidal focusing channel for the beam transport. The specifications of the different components of the drive beam injector can be found in Ref. [10]. Due to the importance of the electron source on the beam quality of the drive beam, our objective here is to find an approach for efficient optimization of the electron source and also the focusing channel. Traditionally one would design such an electron source using extensive particle tracking simulations in a kind of trial and error method. In the previous works $[3,10]$, numerous simulations were done using the 2D ray tracking code EGUN [11], the 2D PIC-code magic [12] and the 3D particle tracking code PARMELA [13] to define the structure of the gun and its associated focusing channel. The simulation results showed that a beam with high quality can be obtained from the gun with $12 \mathrm{~mm}$ mrad normalized rms emittance increasing to $22 \mathrm{~mm} \mathrm{mrad}$ due to the emittance growth during the bunching process. In the present work we plan to propose an analytical approach to first optimize the emittance out of the thermionic gun and its subsequent transport. Using the approach, we design the CLIC thermionic gun and its focusing channel with a significant decrease in the beam emittance generated from the gun and also along the whole injector. The paper is organized as follows. In the next section, we will shortly discuss the basic requirements related to the design of an electron source. Our study covers the beam envelope equation in an axially symmetric situation [14] and the emittance variation formula [15] which provide the basis of the design approach. In Sec. III, 


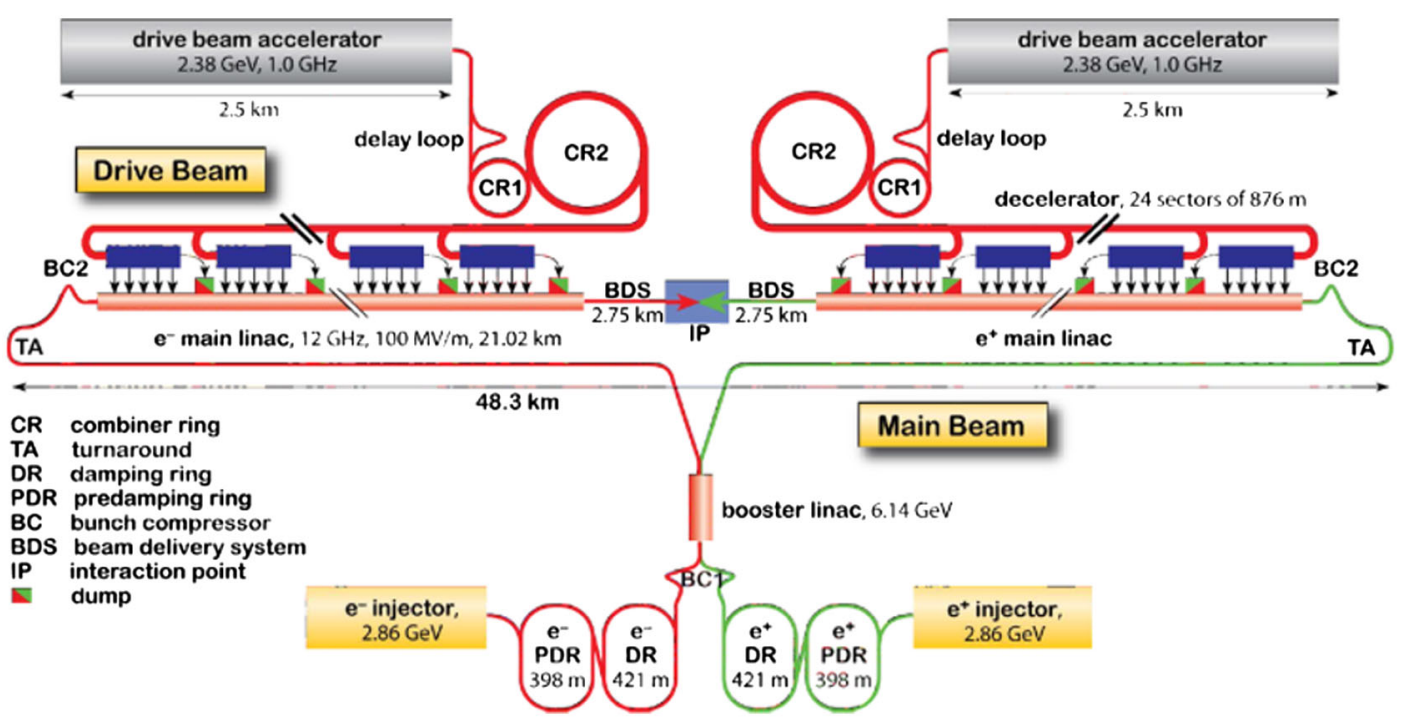

FIG. 1. CLIC layout (not to scale). The main beams are accelerated at the center of the complex using the rf power extracted from the drive beam in the power extraction and transfer structures (PETS blue boxes) [2].

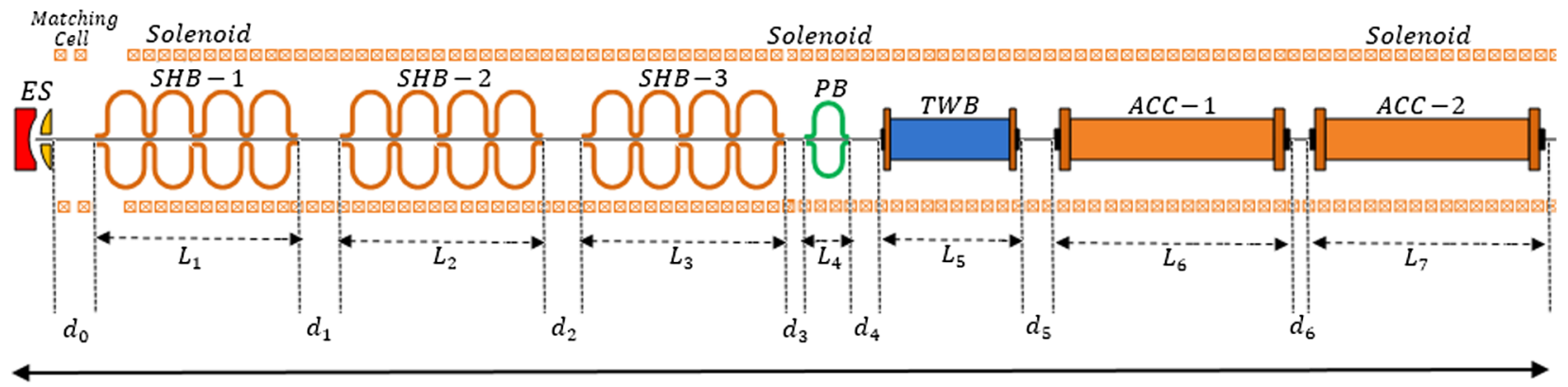

$12.7 \mathrm{~m}$

FIG. 2. CLIC injector layout with a total length of $12.7 \mathrm{~m}$ consists of an electron source (ES), three subharmonic bunchers (SHB), a prebuncher (PB), a traveling wave buncher (TWB) and two accelerating structures (ACC-1,2). A solenoidal focusing channel covers the injector and the accelerating structures.

we present a new analytical approach for the design of a low emittance thermionic gun and apply it for the CLIC drive beam injector. To validate the approach, we also simulate the gun using CST TRACK SOLVER [16]. Section IV is devoted to the transport of the beam downstream of the gun inside a solenoidal focusing channel. We employ our approach based on the beam envelope equation once again to find the optimum structure of the focusing channel. Results of the simulations with PARMELA are presented in this section to confirm the precision of the approach. We finally conclude the paper with a short summary of the findings.

\section{BEAM ENVELOPE EQUATION IN AXIALLY SYMMETRIC FIELDS}

In the following section we introduce the theoretical base that we need for the design of low emittance electron sources and the subsequent focusing channels. We start with the general form of the beam envelope equation for paraxial beams in axially symmetric fields. This relates the variations of the rms beam radius $\sigma=\sqrt{\left\langle x^{2}+y^{2}\right\rangle}$ to the electromagnetic fields, space charge fields and the beam emittance through [14]

$$
\sigma^{\prime \prime}+\frac{\gamma \gamma^{\prime}}{\gamma^{2}-1} \sigma^{\prime}+k_{e m} \sigma-\frac{k_{\varepsilon}}{\sigma^{3}}-\frac{k_{s}}{\sigma}=0
$$

In Eq. (1) it is assumed that the beam propagates in the $z$ direction and the primes denote the derivatives with respect to $z$. The paraxial approximation applies for a beam of particles with the trajectories very close to the beam symmetry axis. In other words $r=\sqrt{x^{2}+y^{2}}$ for each particle in the beam is very small compared to the radii of electrodes, coils, etc. that produce the external electromagnetic fields. Also this implies that the particle velocities in the longitudinal direction are much bigger than their transverse velocities, i.e. $\beta_{x} \ll \beta_{z}$ and $\beta_{y} \ll \beta_{z}$ ( $\beta$ is the 
normalized velocity of the particles to the velocity of light c). In Eq. (1), $\gamma$ is the usual relativistic factor which can be related to the longitudinal component of the electric field on the beam axis $E_{z}$ through

$$
\gamma^{\prime}=\frac{q E_{z}}{m c^{2}}
$$

where $q, m$ and $c$ are the particle charge, mass and speed of light, respectively. Integration of Eq. (2) leads to the energy conservation law. On the other hand, the $k$ terms, i.e. $k_{e m} \sigma$, $\frac{k_{\varepsilon}}{\sigma^{3}}$ and $\frac{k_{s}}{\sigma}$, are known as the effective transverse forces, which act on the beam as a whole and determine the variations of the beam rms radius $\sigma$ along the beam trajectory. The $k_{e m} \sigma$ term shows the effective transverse force of the external electromagnetic fields which include electrostatic, magnetostatic and $\mathrm{rf}$ fields. For paraxial beams the parameter $k_{e m}$ is related to the longitudinal components of the electric $E_{z}$ and magnetic $B_{z}$ fields on the beam axis through

$$
\begin{aligned}
k_{e m}= & \left(\frac{q c B_{z}}{2 m c^{2} \sqrt{\gamma^{2}-1}}\right)^{2}+\frac{q}{2 m c^{2}}\left[\frac{\gamma}{\left(\gamma^{2}-1\right)} \frac{\partial}{\partial z}\right. \\
& \left.+\frac{1}{c \sqrt{\gamma^{2}-1}} \frac{\partial}{\partial t}\right] E_{z} .
\end{aligned}
$$

We note that in Eq. (3), the $\frac{\partial E_{z}}{\partial z}$ and $\frac{\partial E_{z}}{\partial t}$ terms lead to the transverse components of the electric and magnetic fields, respectively [17],

$$
\begin{aligned}
& E_{r}=-\frac{r}{2} \frac{\partial E_{z}}{\partial z}, \\
& B_{r}=+\frac{r}{2 c^{2}} \frac{\partial E_{z}}{\partial t} .
\end{aligned}
$$

Equation (3) shows that the electromagnetic force in the case of the magnetostatic fields has only a focusing effect while for the electrostatic and also rf fields depending on the derivatives of $E_{z}$ with respect to time and $z$, it can be either focusing or defocusing. Beam emittance considerations appear in the beam envelope equation through emittance force $\frac{k_{\varepsilon}}{\sigma^{3}}$. It originates from the random transverse velocities of the particles, which naturally lead to the envelope growth. It has, therefore, only a defocusing effect on the beam. Given the beam emittance definition $\varepsilon_{x}=$ $\sqrt{x^{2} x_{p}{ }^{2}-x x_{p}^{2}}\left(x_{p}=\gamma \beta_{x}\right)$, the parameter $k_{\varepsilon}$ relates to the beam emittance through

$$
k_{\varepsilon}=\frac{4 \varepsilon_{x}^{2}}{\left(\gamma^{2}-1\right)} .
$$

The last term in the beam envelope equation $\frac{k_{s}}{\sigma}$ is known as the space charge force. This force is due to only the electromagnetic fields produced by the beam itself and has a pure defocusing effect on the beam envelope. The parameter $k_{\varepsilon}$ of this force is given by

$$
k_{s}=\frac{q I_{b}}{4 \pi \epsilon_{0} m c^{2} c\left(\gamma^{2}-1\right)^{3 / 2}},
$$

where $I_{b}$ is the average beam current. The general behavior of a beam is determined through the competition between the two last defocusing forces, i.e. the emittance force $\frac{k_{e}}{\sigma^{3}}$ and the space charge force $\frac{k_{s}}{\sigma}$. Depending on which one is larger, the beam is called either an emittance dominated beam or a space charge dominated beam. In the case of the CLIC thermionic gun the space charge force is about 3 orders of magnitude larger than the emittance force and so the beam is totally a space charge dominated beam. For the beam transportation purposes, however, these two forces should be compensated by an appropriate external focusing field. On the other hand, they should be designed in such a way that any emittance growth along the injector could be prevented. In the electron gun, one could achieve this goal by an appropriate design of the focusing electrodes and for downstream of the gun by a series of magnetic coils with appropriate currents and positions. The beam generated in the cathode has a nonvanishing emittance. This is due to the thermal transverse velocity distribution of the particles resulting from the cathode temperature (about $1200^{\circ} \mathrm{C}$ in the case of the CLIC electron gun). On the other hand, downstream of the cathode there are several mechanisms which give rise to the beam emittance growth [14]. In general the variation of the beam emittance is given by the following equation [15]:

$$
\left(\varepsilon_{x}^{2}\right)^{\prime}=\frac{-q \sigma^{2}}{2 I_{b} p_{a v}} \times \frac{\partial}{\partial z}\left[\Delta U_{b}\right],
$$

where $\Delta U_{b}$ is the beam free energy and $p_{a v}$ is the average momentum of the particles. The beam free energy $\Delta U_{b}$ is given by [15]

$$
\Delta U_{b}=\frac{\epsilon_{0}}{2} \int_{0}^{\infty}\left[\left|\beta \vec{E}_{b}\right|^{2}-\left|\beta \vec{E}_{e q}\right|^{2}\right] 2 \pi r d r .
$$

In Eq. (9), $\vec{E}_{b}$ is the electric field resulting from the beam itself and $\vec{E}_{e q}$ denotes the electric field of the equivalent beam. The equivalent beam corresponds to the real beam through having the same energy, the same current and rms radial beam size, but with a uniform distribution. Equation (8) indicates that any nonuniformity in the beam distribution gives rise to the emittance variation, especially for larger rms beam radii and lower energies. It is known in the realm of the beam transport that the linear fields will map a uniform beam to another uniform beam while the nonlinear fields will change the beam uniformity. Therefore, any nonlinearity in the applied fields can change the beam uniformity and result in an emittance growth. On the other 
hand, the space charge fields coming from a nonuniform beam are nonlinear and can directly lead to an emittance growth. Based on the above discussion, our goal in the next section is to design the CLIC electron source by omitting all the nonlinear fields.

\section{DESIGN OF A LOW EMITTANCE ELECTRON GUN FOR THE CLIC DRIVE BEAM INJECTOR}

Any thermionic electron source consists of three important parts: a cathode, a focusing electrode and an anode. The biggest problem in designing such electron guns is to find the optimum geometry of these three parts with the aim of generating a high quality beam. From the physics point of view the geometries of these electrodes are in fact the geometry of the equipotential surfaces of the electrostatic potential function within the gun denoted by $\Phi_{e}(r, z)$. Therefore, to find the optimum geometry of the electrodes it would be enough to find an appropriate potential function $\Phi_{e}(r, z)$ which can meet the desired conditions. Due to the symmetric structure of the thermionic guns, the electric fields within these structures are in axially symmetric forms. From Maxwell's equations, it can be shown that the electrostatic potential of such fields denoted by $\Phi_{e}(r, z)$ could satisfy a series expression as follows [18]:

$$
\Phi_{e}(r, z)=\sum_{n=0}^{\infty} \frac{(-1)^{n}\left(\frac{r}{2}\right)^{2 n}}{(n !)^{2}} \frac{\partial^{2 n}}{\partial z^{2 n}} \Phi_{e}(0, z)
$$

where $\Phi_{e}(0, z)$ indicates the potential function only on the gun symmetry axis $z$. Equation (10) shows knowing the potential function on the gun symmetry axis is sufficient to calculate the potential function $\Phi_{e}(r, z)$ at any other point. The potential function allows then to calculate the corresponding equipotential surfaces which will provide the appropriate geometries of the electrodes. Therefore, the problem of designing an electron gun will be reduced to the problem of calculation of a one-dimensional function $\Phi_{e}(0, z)$. An appropriate choice of the function should provide smooth variations in the beam envelop without any oscillations to avoid emittance growth [14]. Starting from the envelope equation, we substitute the $\gamma$ in Eqs. (1)-(7) through the simple relation $m c^{2}(\gamma-1)=q \Phi_{e}(0, z)$ and rewrite the envelope equation (1) as a second order differential equation for the function $\Phi_{e}(0, z)$ :

$$
\begin{gathered}
\Phi_{e}^{\prime \prime}(0, z)+\frac{2 \sigma^{\prime}}{\sigma} \Phi_{e}^{\prime}(0, z)+\frac{8 m c^{2} \varepsilon_{x}^{2}}{q \sigma^{4}\left(1-\frac{q \Phi_{e}(0, z)}{m c^{2}}\right)} \\
+\frac{I_{b}}{2 \pi \epsilon_{0} c \sigma^{2}\left(1-\frac{q \Phi_{e}(0, z)}{m c^{2}}\right)} \sqrt{\left(1-\frac{q \Phi_{e}(0, z)}{m c^{2}}\right)^{2}-1} \\
-\frac{2 m c^{2} \sigma^{\prime \prime}\left[\left(1-\frac{q \Phi_{e}(0, z)}{m c^{2}}\right)^{2}-1\right]}{q \sigma\left(1-\frac{q \Phi_{e}(0, z)}{m c^{2}}\right)}=0 .
\end{gathered}
$$

This is a second order nonlinear differential equation with respect to the $\Phi_{\mathrm{e}}(0, z)$ for any given function of the rms beam radius $R(z)$. However, according to our previous discussion about the emittance considerations, the $\sigma(z)$ should be a smooth function with respect to $z$ from the cathode to the position of the anode, which we assumed to be at a distance $\mathrm{d}_{0}$ from the cathode. Therefore, a linear decrease with respect to $z$ should be a good approximation for $\sigma(z)$. We consider the following function for $\sigma(z)$ which shows a decrease in the beam envelope from its initial value at $z=0$ on the cathode surface $\frac{r_{c}}{\sqrt{2}}\left(r_{c}\right.$ being the cathode radius) to $\eta$ times this value at the anode position $\left(z=\mathrm{d}_{0}\right)$ :

$$
\sigma(z)=\frac{r_{c}}{\sqrt{2}}\left(1-(1-\eta) \frac{z}{d_{0}}\right) .
$$

In Eq. (12), $\eta$ is a real number between zero and one, which would be fixed by an initial guess, say $1 / 2$. Also $d_{0}$ which shows the distance between the cathode and the anode is a free parameter which needs to be adjusted in such a way that $\Phi_{\mathrm{e}}\left(0, \mathrm{~d}_{0}\right)$ exactly equals the anode potential. For the case of the CLIC thermionic gun, this is equal to $140 \mathrm{kV}$ [1]:

$$
\Phi_{e}\left(0, d_{0}\right)=140 \mathrm{kV} \text {. }
$$

In order to solve Eq. (11), we need at least two boundary conditions. One of these conditions could be the vanishing potential on the cathode surface. The second one could be obtained from the space charge limit condition which implies that the normal component of the electric field on the cathode surface becomes zero [19]. Therefore, the boundary conditions read

$$
\begin{aligned}
& \Phi_{e}(0,0)=0, \\
& \Phi_{e}^{\prime}(0,0)=0 .
\end{aligned}
$$

We have, therefore, a self-consistent system of equations for $\Phi_{\mathrm{e}}(0, z)$ and $\mathrm{d}_{0}$. Unless the value of $\mathrm{d}_{0}$ is identified, the function $\Phi_{\mathrm{e}}(0, z)$ cannot be calculated. On the other hand, only those values of $\mathrm{d}_{0}$ are acceptable that satisfy the expression (13). But for this we in turn need to know $\Phi_{\mathrm{e}}(0, z)$. Here one needs to seek an iterative approach and to start with an initial estimate for $\mathrm{d}_{0}$ to solve Eq. (11) under the conditions (14) and (15) and find the function $\Phi_{\mathrm{e}}(0, z)$. The approach is very fast and one can find the exact values of $\mathrm{d}_{0}$ and $\Phi_{\mathrm{e}}(0, z)$ after a few iterations. However, in order to avoid emittance growth, care must be taken as the radial component of the electric field related to $\Phi_{e}(0, z)$ should show a linear behavior with respect to $r$ as discussed in the previous section. We denote this component by $E_{r}$ and from Eq. (10) one can easily calculate its series representation as 
$E_{r}(r, z)=-\sum_{n=0}^{\infty} \frac{(-1)^{n+1}\left(\frac{r}{2}\right)^{2 n+1}}{(n) ! \times(n+1) !} \frac{\partial^{2 n+2}}{\partial z^{2 n+2}} \Phi_{e}(0, z)$

It is seen from Eq. (16) that for the purpose of having an $E_{r}$ with a linear behavior as a function of the radial component $\mathrm{r}$, the $\Phi_{e}(0, z)$ should be close to a parabola. Therefore, to ensure the field linearity after solving Eq. (11), we should also check whether or not it is close enough to a parabola. Otherwise, we need to modify the function $\sigma(z)$, through changing the value of $\eta$ for instance, and repeat the procedure until its appropriate form is found. Figure 3 shows the solution for the function $\Phi_{e}(0, z)$ which was obtained using this approach for $\eta=\frac{1}{2}$. We used the fourth order Runge-Kutta method [20] to solve Eq. (11). Calculations show that for $\eta=\frac{1}{2}$ and $\mathrm{d}_{0}=51.565 \mathrm{~mm}$ all the conditions could be satisfied (red dashed line). In Fig. 3 we have also drawn a perfect parabola (black dashed line). This shows that our solution is very close to a parabola and the radial component of the resulting electric field would be very close to a linear function of the radial component $r$.

To calculate $\Phi_{e}(r, z)$ from $\Phi_{e}(0, z)$, we should take the derivatives of $\Phi_{e}(0, z)$ with respect to $z$. We can interpolate our numerical solution presented in Fig. 3 and take its derivatives numerically. Figure 4 shows the resulting equipotential surfaces of $\Phi_{e}(r, z)$ obtained from series expansion (10). It is clear that the equipotential surface with zero potential gives us the geometries of the cathode and the focusing electrode while the equipotential surface with $140 \mathrm{keV}$ gives us the anode geometry.

Figure 5 shows the analytical geometry of zero equipotential surface of the cathode plus the focusing electrode surfaces compared with a parabola. Since in practice the cathode of the CLIC thermionic gun has a flat surface with $10 \mathrm{~mm}$ radius [3], we should use an approximation for the zero equipotential surface. However, Fig. 5 shows that an appropriate parabola can be a very good approximation for the analytical geometry of the focusing electrode. Such a parabola has an equation of $r-r_{c}=h_{3} \times\left(\frac{z}{d_{2}}\right)^{2}$ where $r_{c}$ is

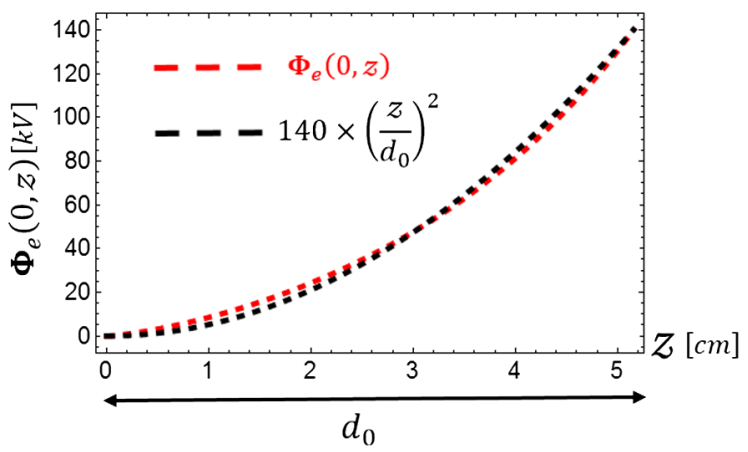

FIG. 3. Numerical solution for the potential equation with linear decrease in the beam envelope to half of its initial value in comparison with a parabola.

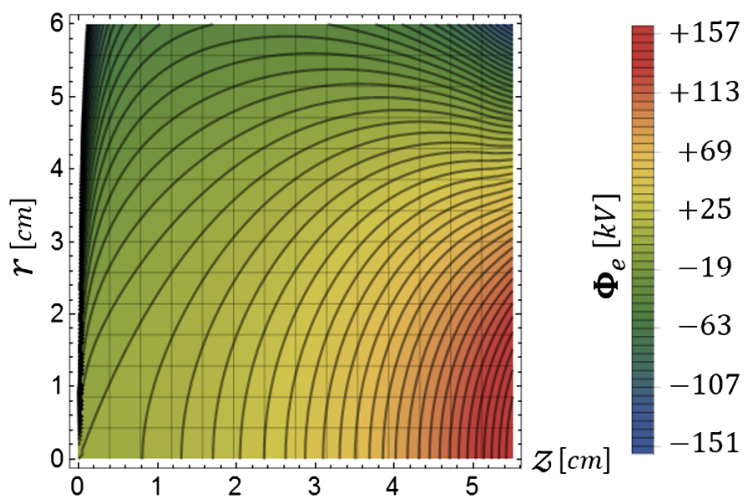

FIG. 4. The equipotential surfaces calculated from series expansion of $\Phi(r, z)$ in terms of $\Phi(0, z)$ and its derivatives.

the cathode radius. $h_{3}$ and $\mathrm{d}_{2}$ are 10,44 and $37 \mathrm{~mm}$, respectively.

As mentioned before the anode should be located at the distance $\mathrm{d}_{0}=51.565 \mathrm{~mm}$ from the cathode. Figure 6 also shows the calculated geometry of the $140 \mathrm{kV}$ equipotential surface (the anode surface) and compares it with a parabola and a simple nose. Since in practice we have to open an aperture in the anode for the beam exit, we should use an approximation for its geometry. Figure 6 shows, however, that an appropriate nose can be a good approximation for the geometry of the anode. Such a nose can be considered as a combination between a curve [with $5 \mathrm{~mm}$ radius $\left(r_{2}\right)$ and $36^{\circ}$ angle $\left(\alpha_{1}\right)$ ], a straight line and a parabola (region marked with hatches). The equation for the parabola is expressed as $z-\mathrm{d}_{0}=\mathrm{d}_{3} \times\left(\frac{r}{h_{4}}\right)^{2}$ where $\mathrm{d}_{0}, \mathrm{~d}_{3}$ and $h_{4}$ are $51.565,35.435$ and $50 \mathrm{~mm}$, respectively.

We note that the important parameter $d_{0}$ is the distance between the vertexes of the analytical curves in Figs. 5 and 6. According to Figs. 5 and 6, our electrode geometries can be defined with seven different parameters: $h_{3}$ and $\mathrm{d}_{2}$ for the cathode geometry, $\mathrm{d}_{3}, h_{4}, \alpha_{1}$ and $h_{5}$ for anode geometry, and $\mathrm{d}_{0}$ for the distance between the nose center and the

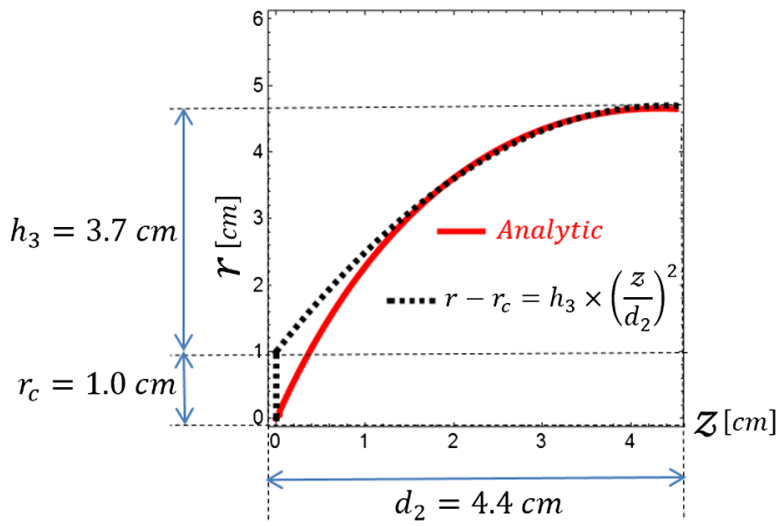

FIG. 5. Geometry of zero equipotential surface (the cathode plus the focusing electrode surfaces) in comparison with a parabola. 


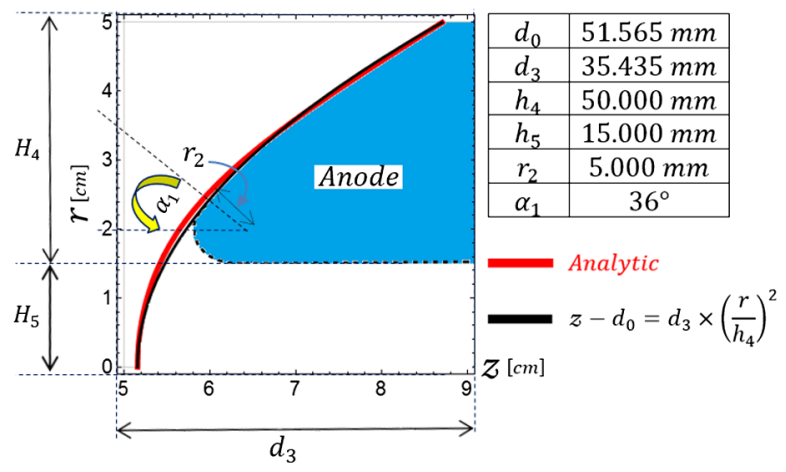

FIG. 6. Analytical geometry of $140 \mathrm{kV}$ equipotential surface (the anode surface) in comparison with a nose (hatched region) composed of a curve, straight line and a part of a parabola. cathode. Our analytical approach for this design determines the starting values for these seven parameters, which can be further optimized depending on the requirements. The final geometry of the CLIC optimized thermionic gun using CST is presented in Fig. 7. The structure has four different parts, namely the cathode and the focusing electrode (both are included in section A in the figure), the anode (section B) and two additional electrodes (sections $\mathrm{C}$ and $\mathrm{D}$ ). The values for all the geometrical parameters shown in the figure are listed on Tables I and II.

The cathode, the central part of the electrode A, has a cylindrical structure with radius $h_{1}=10 \mathrm{~mm}$. As mentioned before the focusing electrode has a parabola shape which is defined with the parameters $h_{3}$ and $\mathrm{d}_{2}$ obtained from our analytical approach with the values of 37 and
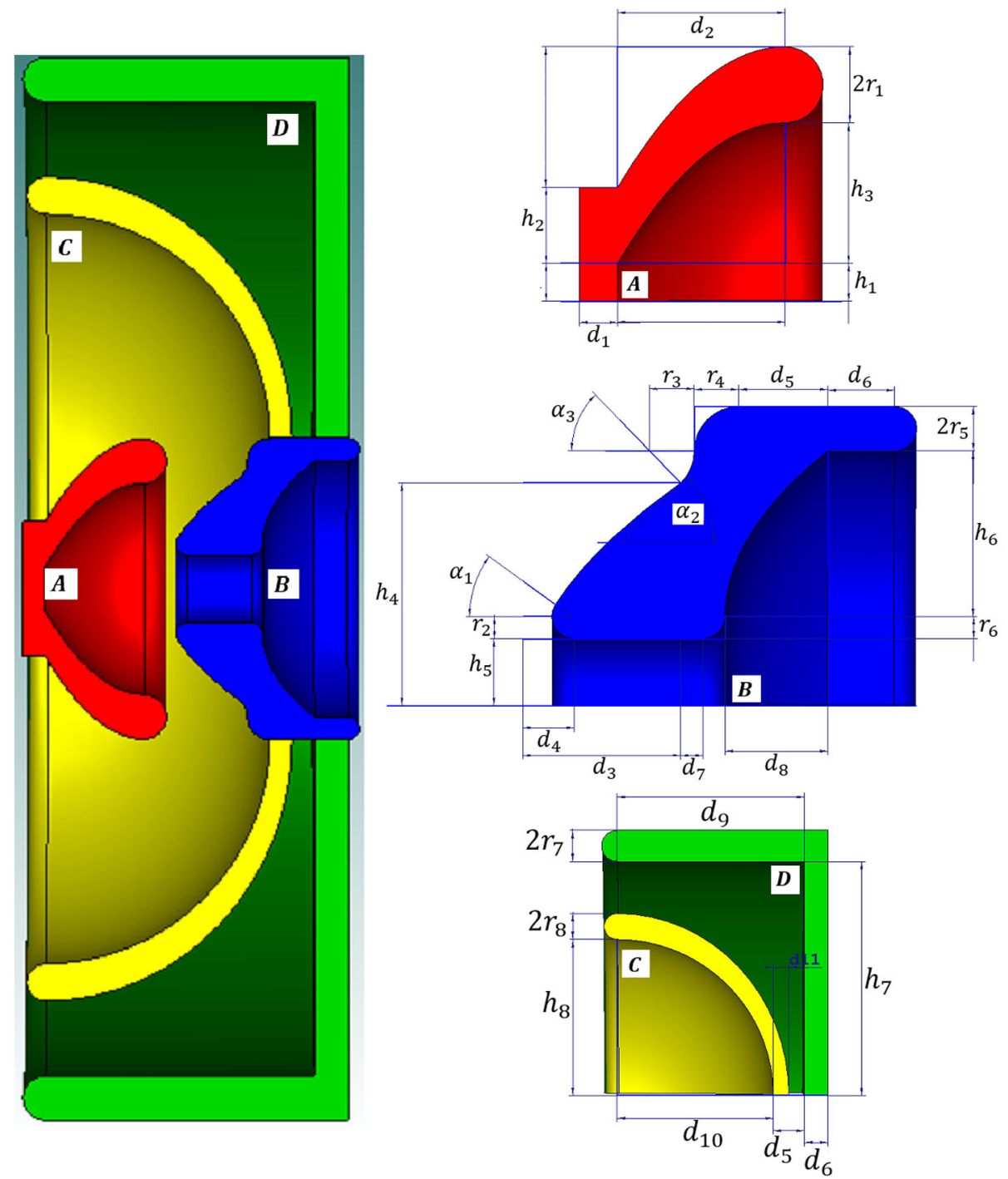

FIG. 7. CST model for the final geometry of the CLIC thermionic gun with all its parameters. Left: the half of the whole structure is presented. Insets, a quarter of each one of the electrodes with all their parameters are shown. Right top: cathode plus focusing electrode; right middle: main part of the anode; right bottom: additional electrodes with the same voltage as the anode. 
TABLE I. The values of the geometrical parameters for the different lengths shown in Fig. 7.

\begin{tabular}{lccr}
\hline \hline Parameter & Size $(\mathrm{mm})$ & Parameter & Size $(\mathrm{mm})$ \\
\hline$h_{1}$ & 10.00 & $\mathrm{~d}_{7}$ & 5.00 \\
$h_{2}$ & 20.00 & $\mathrm{~d}_{8}$ & 23.05 \\
$h_{3}$ & $\mathrm{~d}_{9}$ & 120.05 \\
$h_{4}$ & 37.00 & $\mathrm{~d}_{10}$ & 100.05 \\
$h_{5}$ & 50.00 & $\mathrm{~d}_{11}$ & 10.00 \\
$h_{6}$ & 15.00 & $r_{1}$ & 10.00 \\
$h_{7}$ & 37.20 & $r_{2}$ & 5.00 \\
$h_{8}$ & 150.00 & $r_{3}$ & 10.00 \\
$\mathrm{~d}_{1}$ & 100.00 & $r_{4}$ & 10.00 \\
$\mathrm{~d}_{2}$ & 10.00 & $r_{5}$ & 5.00 \\
$\mathrm{~d}_{3}$ & 44.00 & $r_{6}$ & 5.00 \\
$\mathrm{~d}_{4}$ & 33.44 & $r_{7}$ & 10.00 \\
$\mathrm{~d}_{5}$ & 11.00 & $r_{8}$ & 8.35 \\
$\mathrm{~d}_{6}$ & 20.00 & $\cdots$ & $\cdots$ \\
\hline \hline
\end{tabular}

$44 \mathrm{~mm}$, respectively. The anode has a nose shape with three main parameters, namely $\mathrm{d}_{3}, h_{4}, r_{2}$ with the values of $33.435,50$, and $5 \mathrm{~mm}$, respectively. The structure also has two additional electrodes: $\mathrm{C}$ electrode which is elliptical and $\mathrm{D}$ electrode which is cylindrical (see Fig. 7). The function of these electrodes is to avoid any field penetration behind the anode. The parameters of these two electrodes, however, are not very critical and they do not exert a significant effect on the beam quality. The elliptical electrode provides more possibility to control the beam envelope and make its variations smoother. Simulations also show that without the cylindrical electrode the beam energy will decrease after the anode. This is due to the field penetration behind the anode which decelerates the electrons. In Fig. 8 we have shown the simulation results of CST TRACK SOLVER for the average energy of the electrons passing through the gun. At the end of the gun the electrons will reach the desired energy of $140 \mathrm{keV}$.

Figure 9 shows the simulation results using CST TRACK SOLVER for the beam envelop and the beam emittance in the longitudinal direction along the gun. The smooth behavior of the beam envelope shows the merit of the design which has led to a high-quality beam with very little emittance growth. Our ideal is to design the gun with no emittance growth, but unfortunately, due to nonlinear effects coming from the approximations used for the cathode and the anode geometries, the emittance has some small variations from

TABLE II. The values of the geometrical parameters for the different angles shown in Fig. 7.

\begin{tabular}{lc}
\hline \hline Parameter & Size $(\mathrm{deg})$ \\
\hline$\alpha_{1}$ & 36 \\
$\alpha_{2}$ & 44 \\
$\alpha_{3}$ & 46 \\
\hline \hline
\end{tabular}

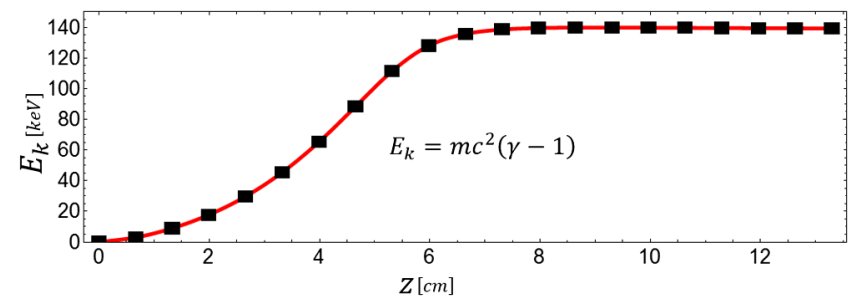

FIG. 8. CST simulation results for variations of the beam energy along the CLIC thermionic gun.
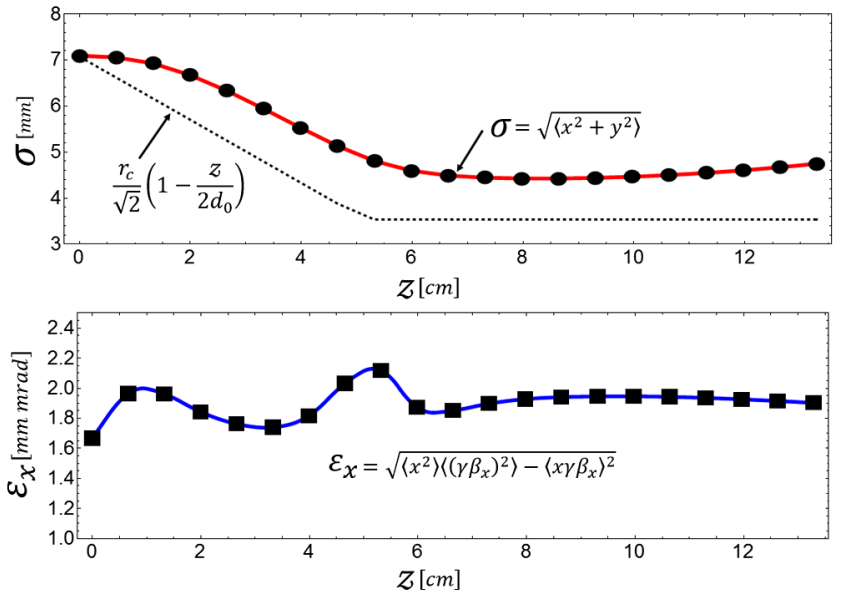

FIG. 9. CST simulation results for the variations of the rms beam radius (top panel) and the beam emittance (bottom panel) along the gun. For the rms beam radius also Eq. (12) is plotted (dashed line).

its thermal value. However, since the field linearity is still very good, the emittance variation is very small and at the end of the gun it does not exceed over $10 \%$ of its intrinsic thermal value. This analytical approach yields an electron source with significant reduced emittance compared with the previous designs with $12 \mathrm{~mm}$ mrad emittance $[3,4]$.

\section{DESIGN OF THE FOCUSING CHANNEL FOR THE CLIC DRIVE BEAM INJECTOR}

To transport the beam after the electron gun we need to make use of a focusing channel. Our focusing channel is a series of $\mathrm{N}$ circular magnetic coils which can produce a longitudinal magnetic field along the beam axis. The focusing channel has two different parts: a matching cell and a solenoid (see Fig. 10).

The first part of the focusing channel, i.e. the matching cell, is composed of two coils with variable positions. On the other hand, the second part, i.e. the solenoidal section, consists of 148 coils located at fixed positions starting from $10 \mathrm{~cm}$ downstream of the beam waist. The waist in turn is $8.6 \mathrm{~cm}$ downstream of the cathode (see Fig. 9). Coils are equally spaced by $10 \mathrm{~cm}$ from each other. All coils have the same radius $\left(a_{c}\right)$, but they are located at different $z_{n}$ positions with respect to the beam waist and carry the 


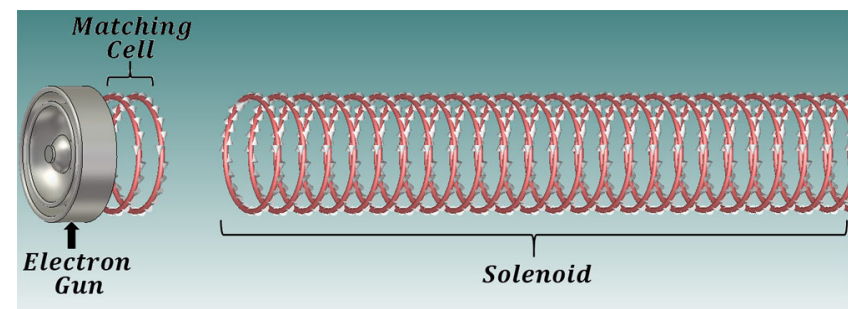

FIG. 10. The complex of the focusing channel. Two matching coils after the electron gun and 148 coils in the solenoidal structure. See also Fig. 2.

currents $I_{n}$. Using the Biot-Savart law [18] for the $z$ component of the induced magnetic field indicated by $B(0, z)$ on the symmetry axis of the focusing channel we can write

$$
B_{z}(0, z)=\sum_{n=1}^{150} \frac{\mu_{0} I_{n}}{2 a_{c} \times\left[1+\left(\frac{z-z_{n}}{a_{c}}\right)^{2}\right]^{\frac{3}{2}}} .
$$

In the matching cell the current and position of the first coil should be calculated in such a way that the magnetic field cannot penetrate back into the gun. This is due to the fact that the presence of the magnetic field in the gun and especially on the cathode surface results in an emittance growth [21]. The current and position of the second coil in the matching cell should be adjusted in such a way that the $R^{\prime}$ and $R^{\prime \prime}$ vanish at the beginning of the solenoid. The adjustment should also reduce the beam rms radius from its initial value at the beam waist $(4.4 \mathrm{~mm})$ to the desired value $(0.5 \mathrm{~mm})$ in the focusing channel. Assuming these conditions are satisfied and the strength of the magnetic field in the solenoid is adjusted appropriately, the beam reaches a thermal equilibrium with an arbitrary constant rms radius (say $0.5 \mathrm{~mm}$ ) within the solenoid. In such a situation we can suppress any oscillations in the beam envelope which could result in an emittance growth and preserve the beam emittance value [14]. The strategy for designing the focusing channel of the CLIC drive beam injector is to maintain the beam rms radius at a constant value say $0.5 \mathrm{~mm}$ along the solenoid. We make use of Eq. (1) with $R^{\prime}=0$ and $R^{\prime \prime}=0$ and obtain the following equation:

$$
\begin{aligned}
\sum_{n=1}^{150} & \frac{\mu_{0} I_{n}}{2 a_{c} \times\left[1+\left(\frac{z-z_{n}}{a_{c}}\right)^{2}\right]^{\frac{3}{2}}} \\
= & \frac{2 m c^{2} \sqrt{\gamma^{2}-1}}{q c}\left\{\frac{q I_{b}}{4 \pi \epsilon_{0} m c^{2} c\left(\gamma^{2}-1\right)^{3 / 2} \sigma^{2}}\right. \\
& +\frac{4 \varepsilon_{x}^{2}}{\left(\langle\gamma\rangle^{2}-1\right) \sigma^{4}}-\left[\frac{q \gamma}{2 m c^{2}\left(\gamma^{2}-1\right)} \frac{\partial}{\partial z}\right. \\
& \left.\left.+\frac{q}{2 m c^{2} c \sqrt{\gamma^{2}-1}} \frac{\partial}{\partial t}\right] E_{z}\right\}^{\frac{1}{2}},
\end{aligned}
$$

where $\left\langle I_{b}\right\rangle$ and $\langle\gamma\rangle$ are functions of $z$ and show the average values of the current and the Lorentz factor for the particle within the bunch at each $z$. If $I(t)$ indicates the instantaneous current of a bunch and $N_{b}$ shows the number of the particles within the bunch, then for the average current $\left\langle I_{b}\right\rangle$ and the average Lorentz factor $\langle\gamma\rangle$ of the bunch we can write

$$
\begin{gathered}
\left\langle I_{b}(z)\right\rangle=\frac{\int_{-\infty}^{+\infty} I(t, z)^{2} d t}{\int_{-\infty}^{+\infty} I(t, z) d t}, \\
\langle\gamma(z)\rangle=\frac{1}{N_{b}} \sum_{i=1}^{N_{b}} \gamma_{i}(z) .
\end{gathered}
$$

Note that for keeping the beam envelope on a constant value $R$, Eq. (18) relates the necessary current $\left(I_{n}\right)$ of the solenoid, to the average bunch current $\left(\left\langle\mathrm{I}_{b}\right\rangle\right)$, the beam emittance $\left(\varepsilon_{x}\right)$ and the electric field $\left(E_{z}\right)$. Given $\left\langle I_{b}\right\rangle, \varepsilon_{x}$ and $E_{z}$ as functions of $z$ along the injector and using Eq. (18), one can solve the system of equations to find the appropriate values of $I_{n}$. Although the functions $E_{z}$ are determined throughout the injector (see Ref. [10]), the current and emittance of the bunches are not predefined and are strongly related to the magnetic field profile of the focusing channel. Therefore, to find the appropriate values of $I_{n}$, we have to follow an iterative approach. We first start with initial guesses for the functions $\left\langle I_{b}\right\rangle$ and $\varepsilon_{x}$. A good starting point could be the assumption of constant values for these two functions along the whole focusing channel, e.g. $\left\langle I_{b}\right\rangle=5 \mathrm{~A}$ and $\varepsilon_{x}=1.8 \mathrm{~mm} \mathrm{mrad}$, which are the values of $\left\langle I_{b}\right\rangle$ and $\varepsilon_{x}$ at the beam waist location. Using a Mathematica [22] program we can find the appropriate set of coil currents to satisfy Eq. (18). Armed with this set, one can solve the envelope equation [Eq. (1)] along the whole focusing channel which may have some oscillation along the solenoid. However, through adjustment of the matching coil parameters $\left(I_{1}, I_{2}\right.$ and $z_{1}$ and $\left.z_{2}\right)$, not only could we cancel the magnetic field at the waist location, but we could also match the beam envelope to the solenoid with no oscillations. Here one reaches the appropriate set of currents and so the required focusing channel structure for the first order of approximation. Later, we use the PIC-code PARMELA to simulate the evolution of the beam all the way down the focusing channel and calculate the functions of the average bunch current and the beam emittance for the second order of approximation. Equipped with these two new functions, we can repeat the procedure to find the currents for the second order of approximation. This procedure can be repeated to any desired order of accuracy until the converged form for the functions $\left\langle I_{b}\right\rangle, \varepsilon_{x}$ are found. The approach is fortunately very fast and just after a few iterations the solutions are obtained. Figure 11 shows the simulation results with PARMELA for the variations of the average bunch energy $\left\langle E_{k}(z)\right\rangle=m c^{2}[\langle\gamma(z)\rangle-1]$ and its current $\left\langle I_{b}\right\rangle$ along the focusing channel. As can be seen from Fig. 11 the average bunch energy and current start to 

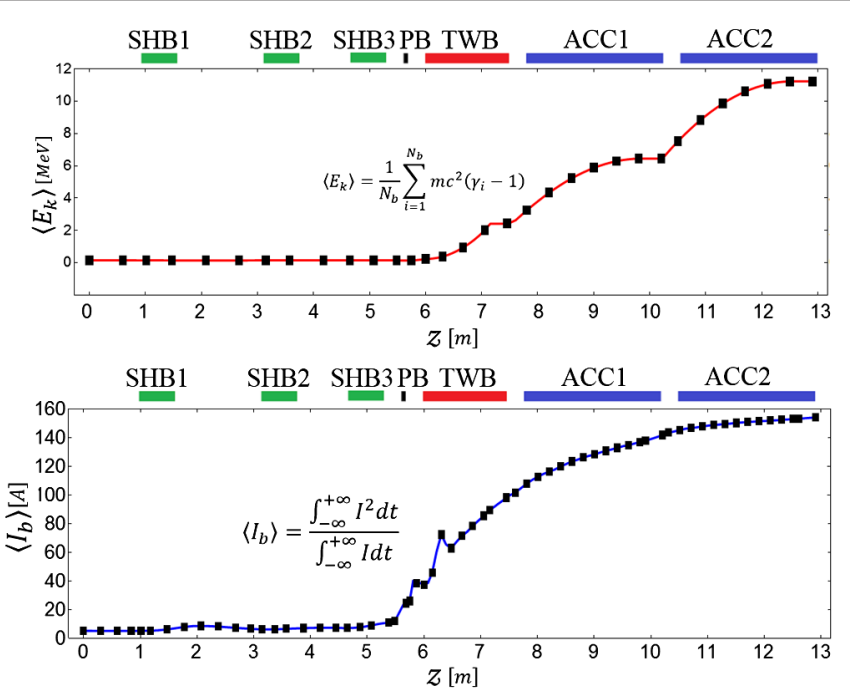

FIG. 11. PARMELA simulation results for the variations of the average bunch energy (top panel) and its current (bottom panel) in the focusing channel designed for the CLIC drive beam injector.

increase very fast from the buncher and reach $11 \mathrm{MeV}$ and $154 \mathrm{~A}$ at the end of the injector. Due to the sharp and significant variations of the two quantities an accurate design for the focusing channel is required.

Figure 12 shows the current distribution of the coils and the magnetic field profile of the designed focusing channel for the drive beam injector calculated by the descripted method. As can be seen at the waist location the magnetic field is exactly zero as it was required for emittance conservation. Also along the buncher where we have a significant increase in the average bunch current and energy, the magnetic field takes its maximum value of $0.5 \mathrm{~T}$ to transport the beam properly. For this structure using the envelope equation (1) we have plotted in Fig. 13
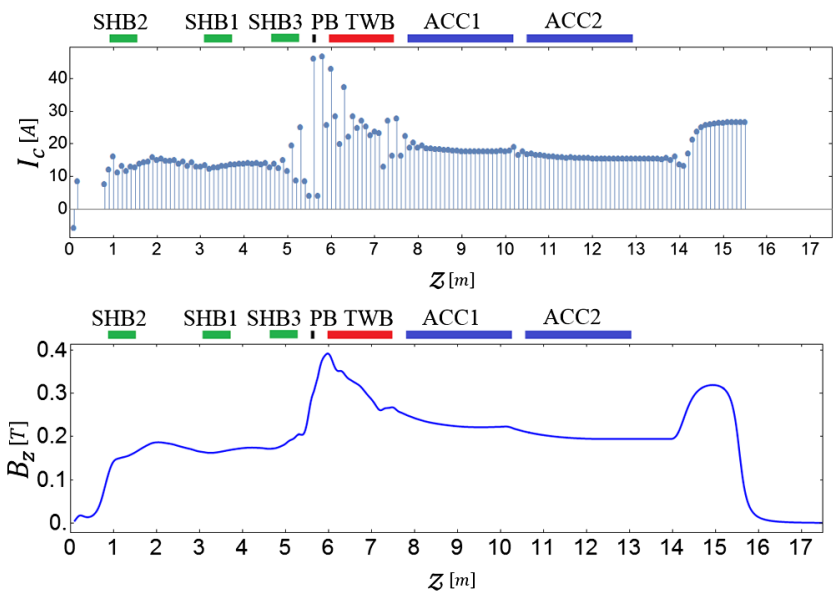

FIG. 12. The current distribution of the magnetic coils (top panel) and the magnetic field profile (bottom panel) in the focusing channel from the beam waist designed for the CLIC drive beam injector.

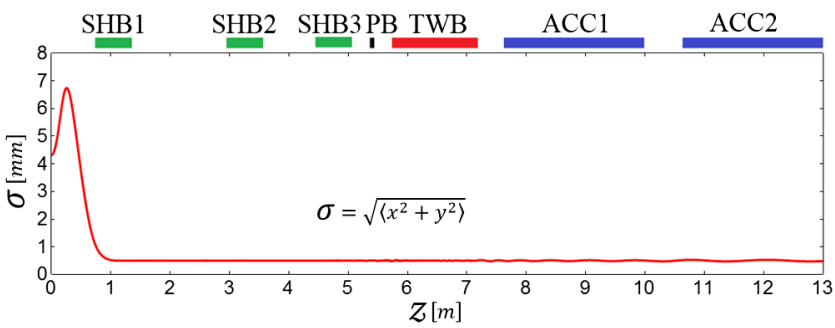

FIG. 13. Variations of the rms beam radius from the beam waist to the end of the injector using the envelope equation.
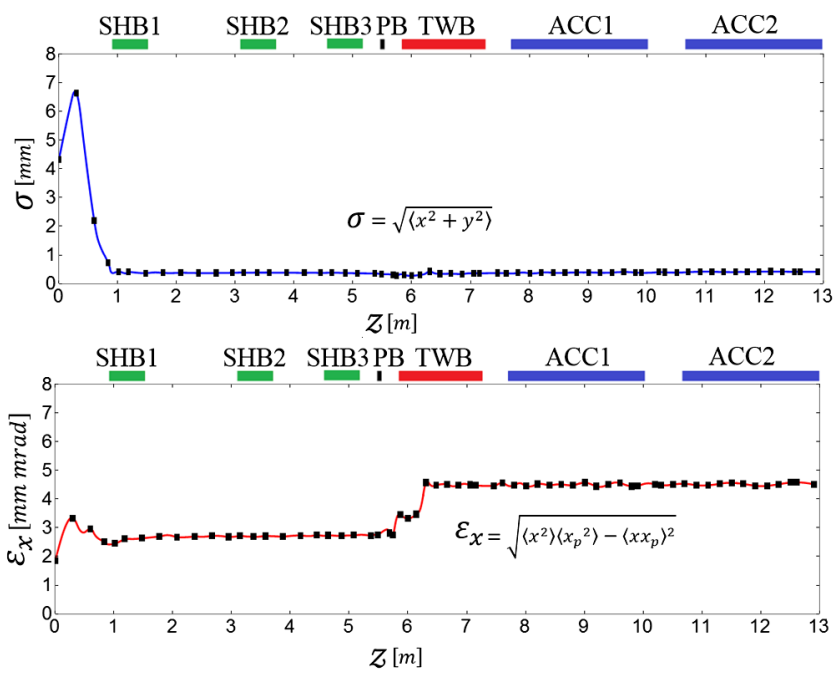

FIG. 14. PARMELA simulation results for the variations of the beam rms radius (top panel) and the beam emittance (bottom panel) within the focusing channel designed for the CLIC drive beam injector.

the variations of the beam rms radius from the beam waist to the end of the injector. The diagram shows that with the designed focusing channel, the beam should be well transported along the whole injector without any oscillation and at a fixed rms radius of $0.5 \mathrm{~mm}$.

Figure 14 demonstrates the variations of the beam envelope and the beam emittance along the whole injector. Figure 13 indicates that the structure found for the focusing channel gives rise to a beam with a constant rms radius $(0.5 \mathrm{~mm})$ and very small emittance $(4.5 \mathrm{~mm} \mathrm{mrad})$ along the whole injector. This shows a significant accomplishment in decreasing the beam emittance along the CLIC drive beam injector in comparison with the previous work with a $22 \mathrm{~mm}$ mrad emittance [10].

\section{CONCLUSIONS AND OUTLOOK}

We have reported the design of the electron source and the focusing channel of the CLIC drive beam injector. We have devised a new analytical approach to design high current thermionic guns and their associated focusing channels. The approach results in small emittances and 
agrees very well with PIC simulations. Our approach takes advantage of the beam envelope equations in axially symmetric fields and the emittance variation formula. The method provides the optimum geometry of different electrodes of the gun and also the structure of the focusing channel for optimal beam transport. We have demonstrated the feasibility of our method with the design of the thermionic gun for the CLIC drive beam injector and its focusing channel. We compared our results with the results obtained by the simulation codes like CST and PARMELA with excellent agreement. The final results greatly improve the beam emittance of the CLIC thermionic gun and the whole CLIC drive beam injector.

[1] A multi-TeV linear collider based on CLIC technology: CLIC conceptual design report, edited by M. Aicheler et al., Report No. CERN-2012-007, 2012.

[2] E. Adli, X-band rf power production, and deceleration in the two-beam test stand of the Compact Linear Collider test facility, Phys. Rev. ST Accel. Beams 14, 081001 (2011).

[3] S. Doebert et al., Design of a high current electron source for the CLIC drive beam injector, in Proceedings of LINAC (Geneva, Switzerland, 2014).

[4] A. D. Yeremian, A. Jensen, E. Jongewaard, and J. Neilson, CLIC drive beam gun Report No. SLAC-Pub-16407, 2015.

[5] M. C. Divall et al., Fast phase switching within the bunch train of the PHIN photoinjector at CERN using fiber-optic modulators on the drive laser, Nucl. Instrum. Methods Phys. Res., Sect. A 659, 1 (2011).

[6] H. Shaker et al., Subharmonic buncher design for the CLIC drive beam injector, in Proceedings of the 4th International Particle Accelerator Conference, IPAC-2013, Shanghai, China, 2013 (JACoW, Shanghai, China, 2013).
[7] M. D. Kelisani et al., Design and beamloading-simulations of a prebunching cavity for the CLIC drive beam injector, in Proceedings of LINAC (Geneva, Switzerland, 2014).

[8] S. H. S. Hajari et al., Beam dynamics studies of the CLIC drive beam injector, in Proceedings of LINAC (Geneva, Switzerland, 2014).

[9] R. Wegner and E. Jensen, CLIC drive beam accelerating structures Report No. CLICNote-945, 2012.

[10] S. H. S. Hajari et al., Beam dynamics studies of the CLIC drive beam injector, in Proceedings of LINAC (Geneva, Switzerland, 2014).

[11] W. B. Hermansfeldt, EGUN an electron optics, and gun design program Report No. SLAC-PUB331, 1988.

[12] B. Goplen, L. Ludeking, D. Smith, and G. Warren, User-configurable MAGIC code for electromagnetic PIC calculations, Comput. Phys. Commun. 87, 54 (1995).

[13] PARMELA, Phase, and radial motion in electron linear accelerators, 2005.

[14] M. Reiser, Theory, and Design of Charged Particle Beams, 2nd ed. (Wiley-VCH Verlag GMBH, Weinheim, 2008).

[15] T. P. Wangler, K. R. Crandall, R. S. Mills, and M. Reiser, Relation between field energy and rms emittance in intense particle beams, IEEE Trans. Nucl. Sci. 32, 2196 (1985).

[16] CST StUdio SuITE 2014, Computer simulation technology.

[17] T. P. Wangler, RF Linear Accelerators, 2nd ed. (John Wiley \& Sons, New York, 2008).

[18] J. D. Jackson, Classical Electrodynamics, 3rd ed. (John Wiley \& Sons, New York, 1999).

[19] S. Humphries, Jr., Charged Particle Beams, 2nd ed. (John Wiley \& Sons, New York, 2002).

[20] G. B. Arfken, Mathematical Methods for Physicists, 7th ed. (Elsevier, New York, 2013).

[21] P. M. Lapostolle and A. L. Septier, Linear Accelerators (North-Holland Publishing Company, Amsterdam, 1970).

[22] Wolfram Research, Inc., Mathematica, Version 10.1, Champaign, IL, 2015. 